International Journal of Computer Science \& Information Technology (IJCSIT) Vol 4, No 2, April 2012

\title{
Embedded Controller for Vehicle In-Front Obstacle Detection and Cabin Safety Alert System
}

\author{
V.Ramya ${ }^{1}$, B. Palaniappan ${ }^{2}$, K. Karthick ${ }^{3}$ \\ ${ }^{1}$-Assistant Professor, Dept of CSE, Annamalai University, Chidambaram, India. \\ E-Mail: ramyshrieyahoo.com \\ ${ }^{2}$-Dean, FEAT, Head, Dept of CSE, Annamalai University, Chidambaram, India. \\ E-Mail: bpau2002@yahoo.co.in \\ 3 - B.E [I.T], Dept of CSE, Annamalai University, Chidambaram, India. \\ E-Mail: kkpsslvegmail.com
}

\begin{abstract}
In today's world safety and security plays an important role, hence we tend to provide a good safety and security system while travelling. Vehicles are important in today's fast-paced society. Hence, acquiring a vehicle nowadays is considered a necessity, compared to the past where it was considered a luxury. In this thriving society, more and more vehicles are produced to meet the increasing demands of people and businesses from all corners of the world. Here comes the necessity to provide more and more safety and security features to them. Hence this project aims to design an embedded system for vehicle cabin safety and security by modifying and integrating the existing modules. This monitors the level of the toxic gases such as CO, LPG and alcohol inside the vehicle and provides alert information in the form of alarm during the critical situations. And also send SMS to the authorised person through the GSM. An IR Sensor is used to detect the static obstacle in front of the vehicle and the vehicle gets stopped if any obstacle is detected. This may avoid accidents due to collision of vehicles with any static obstacles.
\end{abstract}

Keywords: ADC, GSM, Embedded System, Microcontroller, Sensors.

\section{INTRODUCTION}

Vehicle is the first place where safety starts. Hence we must try to equip it with the latest technologies and measures to make it a safe machine and also to keep our self and our loved ones safe. Always remember that safety starts and ends with the person who drives the vehicle. The cars and trucks found today have high range of inbuilt safety accessories to protect their passengers. Before it used to be just seatbelts, but now more features have been included which are more advanced and efficient than seatbelts. Warning alerts and alarms are other security systems incorporated in the cars and trucks to alert us about various factors like exceeding speed limit or smoke alarms. These are designed to make the passengers aware of crossing the limitations which is important in most of the time and in most cases. In the same way here an embedded system has been designed to make the journey of the passengers inside a vehicle safe and secure with various recently found safety and security measures.

\section{RELATED WORK}

Wakamatsu, Kitakyushu, Hibikinoetel [1] proposed a new approach on Accident prevention system based on semantic network, here they used the web mining technique to analyse the web information by means of searching, recommending, surfing and visualising the web. The objective of this paper is to build an accident prevention system by means of the semantic 
International Journal of Computer Science \& Information Technology (IJCSIT) Vol 4, No 2, April 2012

network. The system is built from a number of ranking algorithms based on generality and novelty measures extracted from an accident database.

P L Needham et el[2] have used Accident Data Recorders (ADR's) in the area of commercial and public fleets have proved to have a considerable accident preventative effect and as a result have significantly reduced consequential costs. Apart from offering effective relief to professional drivers from the risk of erroneous liability the ADR has served well as a management tool to reduce collisions, damages and repair costs in an unexpected way.

BaoRong Chang, Chung-Ping Young, Hsiu Fen Tsai, and Jian-JrLinet el [3] made study to explore how to realize high performance collision warning system (CWS), providing the precaution against traffic crash in transit. An embedded hybrid adaptive network-based fuzzy inference system (ANFIS) plus quantum-tuned back-propagation neural network (QTBPNN) built anthem platform with Davinci+XScale-NAV270 was employed to realize collision warning system and they also installed motor vehicle event data recorder (MVEDR).

Kai-Tai Song and Chih-Chieh Yang et el [4] have given a design and implementation of a real-time visual tracking system for vehicle safety applications. A novel feature-based vehicle tracking algorithm is proposed. This algorithm can automatically detect and track multiple moving objects, including cars and motorcycles, ahead of the tracking vehicle. Combined with the concept of focus of expansion (FOE) and scene analysis, the developed system can segment features of moving objects from moving background and provide a collision warning in real time. The proposed algorithm is realized using a CMOS image sensor and NMOS embedded processor architecture. The constructed stand-alone visual tracking system has been validated in actual road tests. Experimental results show that the proposed system successfully tracks front vehicles and provides information of collision warning in urban artery with speed around 60 $\mathrm{km} / \mathrm{hr}$. both at night and day times.

PadminiKumari j, S.S.Dorle, A.G.KeskarMegha, B.Chakole et el[5],have presented a method to implement vehicle safety systems with one or two channels and using a single transceiver, micro-controller 89C51 is used as main driver. Emerging systems for active vehicle safety use short range sensors with LOS links, usually to detect vehicles or lane boundaries immediately adjacent to the host vehicle. Typical applications include forward collision warning, adaptive cruise control, and lane keeping.

Soichi Kubota, Yoshiharu Okamoto, Hideo Oda et el [6] have made a report on an experimental system that senses the presence of pedestrians as well as their position, then sends the data to an on-board device which alerts the driver. Additionally, they describe an intervehicle multi-hop broadcasting transmission system in which each vehicle includes a repeater function for provision of the alert information to vehicles further away [6].

LIU Zhen-ya, WANG Zhen-dong and CHEN Rong et el [12] have focused on, Intelligent residential burglar alarm, emergency alarm, fire alarm, toxic gas leakage remote automatic sound alarm and remote control system, which is based on 89C51 single chip computer. The system can be automatic alarm, automatic calling the police hotline number. It can be used voice alarm and show alarm occurred address. It can set up and modify user password. It can be recordable and voice suggestion. It can be used telephone remote control electrical power.

Chen Peijiang and Jiang Xuehuaet el [11] have focused on the GSM, because the wireless remote monitoring system has more and more application, a remote monitoring system based on SMS of GSM is presented. Based on the total design of the system, the hardware and software of the system is designed. In this system, GSM network is a medium for transmitting 
International Journal of Computer Science \& Information Technology (IJCSIT) Vol 4, No 2, April 2012

the remote signal. The system includes two parts which are the monitoring centre and the remote monitoring station. The monitoring centre consists of a computer and a TC35 communication module of GSM. The computer and TC35 are connected by RS232. The remote monitoring station includes a TC35 communication module of GSM, a MSP430F149 MCU, a display unit, various sensors, data gathering and processing unit. The software of the monitoring centre and the remote monitoring station is designed by using VB. The result of demonstration shows that the system can monitor and control the remote communication between the monitoring centre and the remote monitoring station, and the remote monitoring function is realized.

IoanLita, Ion BogdanCioc and Daniel AlexandruVisanet el [15] have presented a low cost automotive localization system using GPS and GSM-SMS services. The system permits localization of the automobile and transmitting the position to the owner on his mobile phone as a short message (SMS) at his request. The system can be interconnected with the car alarm system and alert the owner, on his mobile phone, about the events that occurs with his car when it is parked. The system is composed by a GPS receiver, a microcontroller and a GSM phone. Additional, the system can be settled for acquiring and transmitting of information, when requested, about automobile status and parameters (engine status, speed, direction, etc.) or alert when it started engine, exceed a given speed limit or if leave a specific area. By using the PC connection, the system can be used as navigation system. Optional, the system can be used as car tracking system if connected with GSM/GPRS phone. The presented application is a low cost solution for automobile position localizing and status, very useful in case of car theft situations (alarm alert, engine starting, localizing), for adolescent drivers watching and monitoring by their parents (speed limit exceeding, leaving a specific area), as well as in car tracking system application. The proposed solution can be used in other types of application, where the information needed are requested rarely and at irregular period of time (when requested).

K. Galatsis, W. Wlodarsla, K. Kalantar-Zadeh and A. Trinchiet el [17] have given an idea where the car cabin air quality monitoring can effectively he analysed using metal oxide semiconducting (MOS) gas sensors. In this paper, commercially available gas sensors are compared with fabricated Mo03-Ti02 and Moo3- WO3 thin films. The laboratory tests showed that the Moo3 based sensors possessed comparable gas sensing properties. The Mo03 (75\%)Ti02 (25\%) sensor has a response $74 \%$ higher relative to the hest commercial sensor tested.

K. Galatsis, W. Wlodarski, Y.x. Li and K. Kalantar-zadeh et el[20]have upgraded the idea where the vehicle cabin air quality monitor using carbon monoxide (CO) and oxygen (02) gas sensors has been designed, developed and on-road tested. The continuous monitoring of oxygen and carbon monoxide provides added vehicle safety as alarms could be set off when dangerous gas concentrations are reached, preventing driver fatigue, drowsiness, and exhaust gas suicides. CO concentrations of $30 \mathrm{ppm}$ and oxygen levels lower than $19.5 \%$ were experienced whilst driving [20].

\section{HARDWARE DESCRIPTION}

In our proposed system an obstacle sensor i.e. the IR module is mounted in front the car, such that the distance between the obstacles in front of vehicle is normally of $3 \mathrm{ft}$. is sensed by the sensor module and instructs the microcontroller about the obstacles ahead. The microcontroller then alerts the driver with an alarm and controls the vehicle by stopping it. In the same way a gas sensor is mounted inside the vehicle such that it senses the presence of the gases inside the vehicle cabin and informs the microcontroller if there is any increase in the level of the toxic gases. The microcontroller then alerts the passengers and the driver inside the vehicle with an alarm and also sends a SMS to the authorised user through GSM. 
The block diagram of the proposed system is shown in the fig. 1. The proposed system is developed using Atmel AT89S52 microcontroller. The system has two modules namely the Gas sensing module and the Obstacle detection module and they are interfaced with the microcontroller. An ADC is provided in these two modules for converting the analog acquired data in to the digital data for processing by the microcontroller. The GSM module is connected with the microcontroller through RS232 and the alarm is interfaced with the microcontroller to raise an alarm during the critical situations.

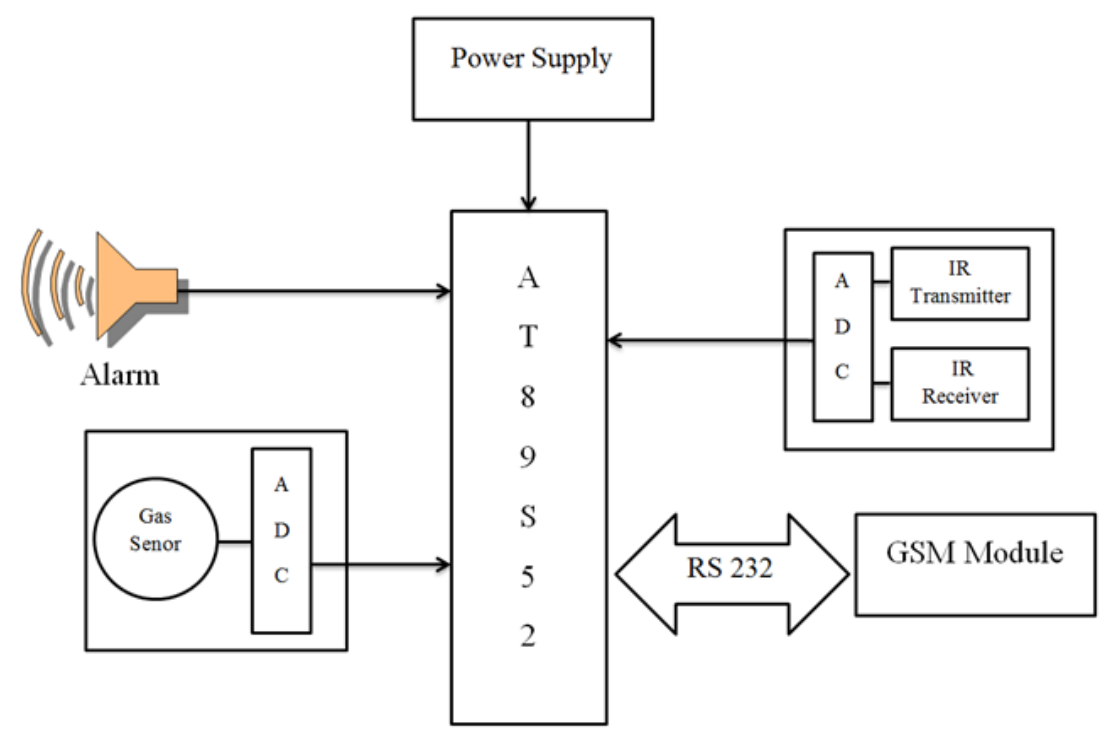

Fig. 1 Block Diagram of the Proposed System

\subsection{The Gas Sensing Module}

The gas sensing module is used to sense the presence of toxic gases such as CO, LPG, Alcohol and other toxic gases inside the vehicle. If critical levels of gases were found, that is if the CO exceeds 20ppm and the level of LPG exceeds 10,000ppm and the presence of alcohol is detected then the digital data from the gas sensing module is sent to the microcontroller which displays the information about the gas leakage inside the vehicle and produces an alarm to alert the persons inside the vehicle. It also sends a text message to the authorised person through the GSM modem connected to the microcontroller such that remedy measures could be taken by the authorised person and to give proper medical treatment to them if required [10].

\subsubsection{Gas Sensors}

A gas detector is a device which detects the presence of various gases within an area, usually as part of a system to warn about gases which might be harmful to humans or animals. Gas detectors can be used to detect combustible, flammable and toxic gases, and also oxygen depletion [10]. This type of device is used widely in industry and can found in a variety of locations such as on oil rigs, to monitor the manufacturing processes and in emerging technologies such as photovoltaic. They may also be used in fire fighting and the gas detectors are usually battery operated. The architecture of the gas sensing module is shown in fig. 2 


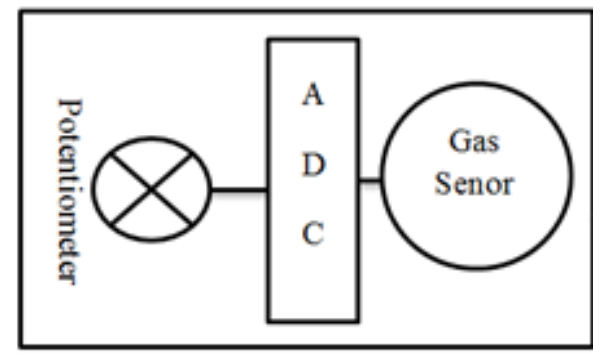

Fig.2Architecture of Gas Sensing Module

The sensing module transmits warnings via a series of audible and visible signals such as alarms and flashing lights, when dangerous levels of gas vapours are detected. As detectors measure a gas concentration, the sensor responds to a calibration gas, which serves as the reference point or scale. As a sensor's detection exceeds a pre-set alarm level, the alarm or signal will be activated. As units, gas detectors are produced as portable or stationary devices. Originally, detectors were produced to detect a single gas, but modern units may detect several toxic or combustible gases, or even a combination of both types.

\subsubsection{MQ-7- Gas Sensor}

A MQ-7 gas sensor is used in this system which is a device that detects the presence of the carbon monoxide (CO) and LPG and various other gases in the environment. CO is a colourless and odourless compound produced by incomplete combustion. It is often referred to as the "silent killer" because it is virtually undetectable without using detection technology. Elevated levels of $\mathrm{CO}$ can be dangerous to humans depending on the amount present and length of exposure. Smaller concentrations can be harmful over longer periods of time while increasing concentrations require diminishing exposure times to be harmful. MQ-7 sensors are designed to measure $\mathrm{CO}$ levels over a time and sounds an alarm before dangerous levels of $\mathrm{CO}$ accumulate in an environment, and therefore giving the people adequate warning to safely ventilate the area or evacuate. Some system-connected detectors also alert a monitoring service that can dispatch emergency services if necessary. The interfacing of the sensor with the microcontroller and the developed gas sensor module is shown in the fig 3(a) and fig 3(b).

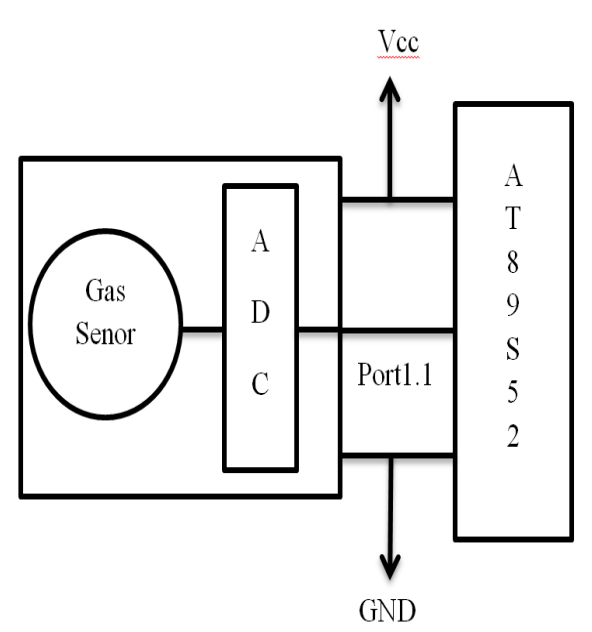

Fig.3(a)

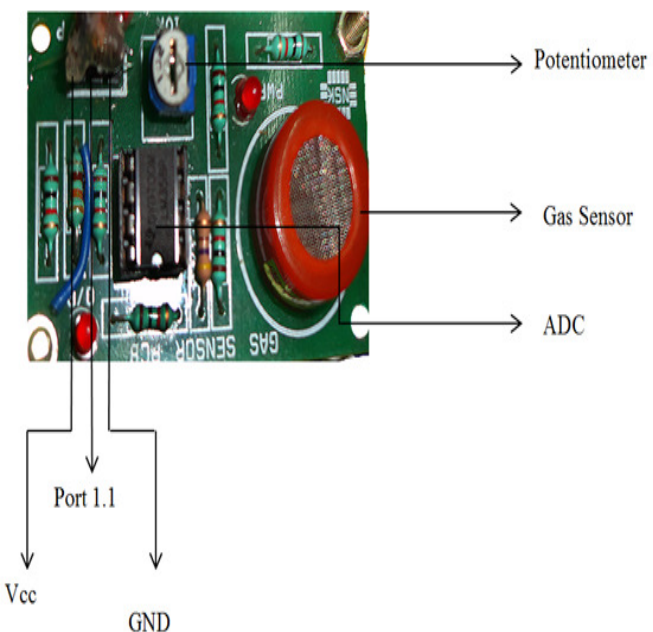

Fig.3(b)

Fig.3(a)Interfacing Gas Sensor with Microcontroller.(b)The Developed Gas sensor module 
International Journal of Computer Science \& Information Technology (IJCSIT) Vol 4, No 2, April 2012

The MQ-7 sensor also detects the presence of a dangerous LPG leak in our home or in a service station, storage tank environment and even in vehicle which uses LPG gas as its fuel. This unit can be easily incorporated into an alarm unit, to sound an alarm or provide a visual indication of the LPG concentration. The sensor has excellent sensitivity combined with a quick response time. When the target combustible gas exist, the sensor's conductivity is higher along with the gas concentration rising. A simple electronic circuit is used to convert the change in conductivity to its corresponding output signal of gas concentration. This module may be used in various application areas such as in Home, Industry and in car [17].

The features of this sensor are,

- High sensitivity to carbon monoxide, LPG, propane etc.,

- Stable and long life

\subsection{Obstacle Sensing Module}

The obstacle sensing module is used to sense the static obstacles in front of the vehicle such that, accidents due to unwanted parking of the vehicles and collision with trees and other objects especially during the night time could be avoided. These obstacles could be detected using various methods such as ultrasonic sensors etc. [6].The working principle of the obstacle sensor is shown in the fig. 4 .

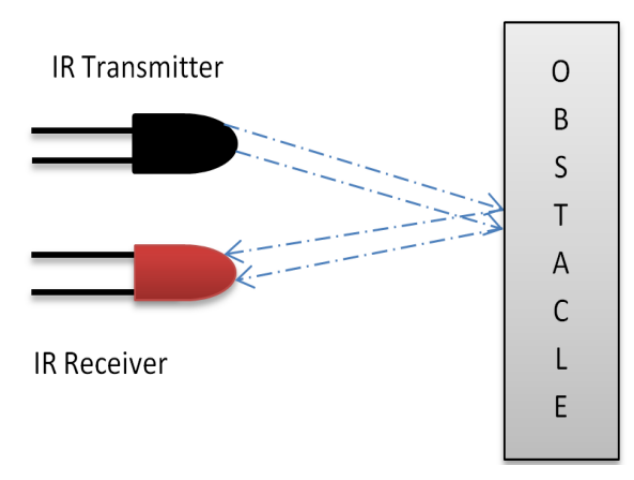

Fig. 4 IR Sensing Module

An IR module is used to detect the vehicle in front obstacles, it consists of an IR transmitter and a Receiver which combines to form an obstacle sensing module. The architecture of IR module is shown in fig. 5.

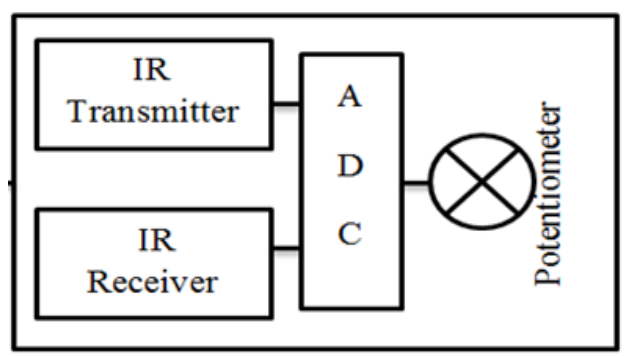

Fig.5 Architecture of the IR module 
International Journal of Computer Science \& Information Technology (IJCSIT) Vol 4, No 2, April 2012

\subsubsection{IR Transmitter}

In general, the basic building block of any IR transmitter is modulation of the information signal with carrier signal, because the receiver modules which are available off-the-shelf are made for a particular carrier frequency. So it is clear that when we choose a particular IR receiver module, we also need to transmit the modulated wave with the same carrier frequency of an IR receiver module.

\subsubsection{IR Receiver}

It is quite simple to construct an IR receiver with readily available off-the-shelf modules. These modules are nothing but the IC packages, referred as TSOP (Thin small-outline package). In this work, the receiver is designed for $38 \mathrm{kHz}$ carrier signal; hence the selected IC should work for the same frequency. The IC TSOP4838 will serve as a receiver module, which is compatible with both TTL and CMOS logic. This means that we can directly get digital signal from the receiver module and then connect it to the microcontroller. The interfacing of IR module with the microcontroller and the developed module is shown in the fig. 6(a) and fig6(b).

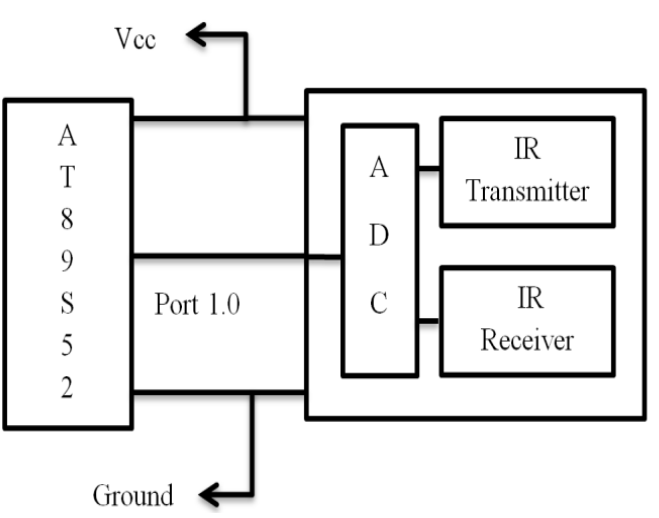

Fig.6(a)

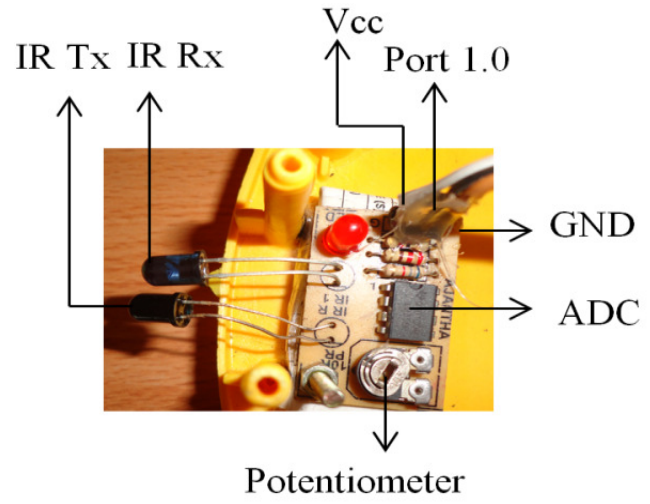

Fig.6(b)

Fig.6 (a) Interfacing IR sensor with AT89S52.(b) The developed Obstacle detection module

\subsection{Microcontroller}

The microcontroller forms the heart of an embedded system. The AT89S52 is the microcontroller used here which is a low-power, high-performance CMOS 8-bit microcontroller with $8 \mathrm{~K}$ bytes of in-system programmable Flash memory. The device is manufactured using Atmel's high-density non-volatile memory technology and is compatible with the industry-standard 80C51 instruction set and pinout. The on-chip Flash allows the program memory to be reprogrammed in-system or by a conventional non-volatile memory programmer. By combining a versatile 8-bit CPU with in-system programmable Flash on a monolithic chip, the Atmel AT89S52 is a powerful microcontroller which provides a highlyflexible and cost-effective solution to many embedded control applications. The AT89S52 provides the following standard features: $8 \mathrm{~K}$ bytes of Flash, 256 bytes of RAM, 32 I/O lines, 
Watchdog timer, two data pointers, three 16-bit timer/counters, a six-vector two-level interrupt architecture, a full duplex serial port, on-chip oscillator, and clock circuitry. In addition, the AT89S52 is designed with static logic for operation down to zero frequency and supports two software selectable power saving modes.

The pin diagram of the AT89S52 is shown in the fig. 7. The microcontroller is always in the active mode, if the gas sensor senses a gas and founds a critical situation then the information is sent to the microcontroller. The microcontroller first provokes an alarm to alert the passengers inside the vehicle and also an alert message to the authorised user is sent in the form of SMS through GSM. The microcontroller uses AT+CMGS command to send the SMS where the GSM is connected to the microcontroller through RS232 cable.

\begin{tabular}{|c|c|c|c|}
\hline & & & \\
\hline (T2) P1.0 & 1 & 40 & V VCC \\
\hline (T2 EX) P1.1 & 2 & 39 & $\mathrm{P} 0.0$ (AD0) \\
\hline P1.2 & 3 & 38 & $\checkmark \mathrm{P} 0.1$ (AD1) \\
\hline P1.3 & 4 & 37 & P0.2 (AD2) \\
\hline P1.4 & 5 & 36 & P0.3 (AD3) \\
\hline (MOSI) P1.5 & 6 & 35 & 7 P0.4 (AD4) \\
\hline (MISO) P1.6 & 7 & 34 & $\checkmark$ P0.5 (AD5) \\
\hline (SCK) P1.7 & 8 & 33 & P0.6 (AD6) \\
\hline RST & 9 & 32 & P0.7 (AD7) \\
\hline (RXD) P3.0 & 10 & 31 & $\overline{\overline{E A}} \mathrm{~V} V P P$ \\
\hline (TXD) P3.1 & 11 & 30 & ALE/PROG \\
\hline 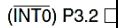 & 12 & 29 & $\overline{\mathrm{PSEN}}$ \\
\hline 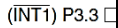 & 13 & 28 & $\checkmark \mathrm{P} 2.7$ (A15) \\
\hline (T0) P3.4 & 14 & 27 & $\neg$ P2.6 (A14) \\
\hline (T1) P3.5 & 15 & 26 & P2.5 (A13) \\
\hline$(\overline{W R})$ P 3.6 & 16 & 25 & $\neg$ P2.4 (A12) \\
\hline 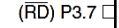 & 17 & 24 & $\neg$ P2.3 (A11) \\
\hline XTAL2 L & 18 & 23 & $\checkmark$ P2.2 (A10) \\
\hline XTAL1 С & 19 & 22 & P2.1 (A9) \\
\hline GND [ & 20 & 21 & P2.0 (A8) \\
\hline
\end{tabular}

Fig. 7 Pin Description of the AT89S52

\subsection{GSM Module}

A GSM modem is a specialized type of modem which accepts a SIM card, and operates over a subscription to a mobile operator, just like a mobile phone. From the mobile operator perspective, a GSM modem looks just like a mobile phone. A GSM modem exposes an interface that allows applications such as SMS to send and receive messages over the modem interface. The mobile operator charges for this message sending and receiving as if it was performed directly on a mobile phone. To perform these tasks, a GSM modem must support an "extended AT command set" for sending/receiving SMS messages, as defined in the ETSI GSM 07.05 and 3GPP TS 27.005 specifications. Due to some compatibility issues that can exist with mobile phones, using a dedicated GSM modem is usually preferable to a GSM mobile phone. It should also be noted that not all phones support the modem interface for sending and receiving SMS messages. In particular, most smart phones, including Blackberries, iPhone, and Windows Mobile devices, do not support this GSM modem interface for sending and receiving SMS messages at all. Additionally, Nokia phones that use the S60 (Series 60) interface, which is Symbian based, only support sending SMS messages via the modem interface, and do not support receiving SMS via the modem interface [15]. The interfacing between the GSM and the Microcontroller and the developed module is shown in the fig. 8(a) and fig.8(b). 


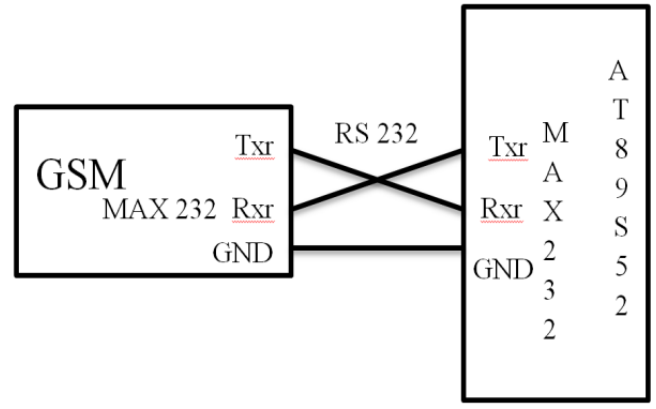

Fig.8(a)

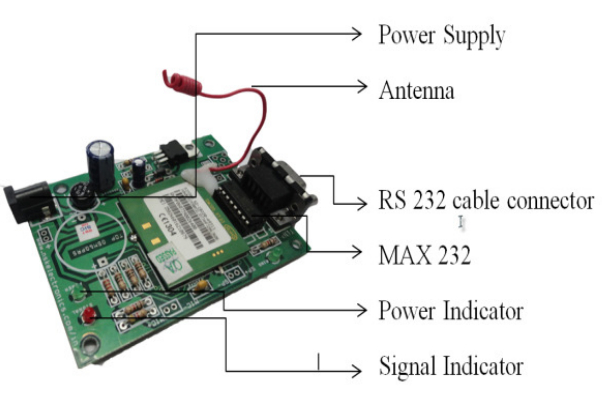

Fig.8(b)

Fig.8(a) Interfacing GSM with AT89S52.(b) The developed GSM module

\section{SOFTWARE DESCRIPTION}

\subsection{Embedded C}

An embedded system is the one which is designed to perform a specific task and the embedded software rules the entire system. This software for a particular embedded system could be developed using various embedded programming languages. But embedded $\mathrm{C}$ is the well-known embedded programming language.

Use of $\mathrm{C}$ in embedded systems is driven by following advantages

- It is small and reasonably simpler to learn, understand, program and debug.

- C Compilers are available for almost all embedded devices in use today, and there is a large pool of experienced $\mathrm{C}$ programmers.

- Unlike assembly, $\mathrm{C}$ has advantage of processor-independence and is not specific to any particular microprocessor/ microcontroller or any system. This makes it convenient for a user to develop programs that can run on most of the systems.

- As C combines functionality of assembly language and features of high level languages, $\mathrm{C}$ is treated as a 'middle-level computer language' or 'high level assembly language'.

- It is fairly efficient.

- It supports access to $\mathrm{I} / \mathrm{O}$ and provides ease of management of large embedded projects.

Many of these advantages are offered by other languages also, but what sets $\mathrm{C}$ apart from others like Pascal, FORTRAN, etc. is the fact that it is a middle level language; it provides direct hardware control without sacrificing benefits of high level languages. Compared to other high level languages, $\mathrm{C}$ offers more flexibility because $\mathrm{C}$ is relatively small, structured language; it supports low-level bit-wise data manipulation. Compared to assembly language, $\mathrm{C}$ Code written is more reliable and scalable, more portable between different platforms (with some changes) [13]. Moreover, programs developed in $\mathrm{C}$ are much easier to understand, maintain and debug. Also, as they can be developed more quickly, codes written in $\mathrm{C}$ offers better productivity. $\mathrm{C}$ is based on the philosophy 'programmers know what they are doing'; only the intentions are to be stated explicitly. It is easier to write good code in $\mathrm{C}$ and convert it to an efficient assembly code (using high quality compilers) rather than writing an efficient 
International Journal of Computer Science \& Information Technology (IJCSIT) Vol 4, No 2, April 2012

code in assembly itself. Benefits of assembly language programming over $\mathrm{C}$ are negligible when we compare the ease with which $\mathrm{C}$ programs are developed by programmers.

\subsection{Keil Compiler}

After developing the software, it must be downloaded to the microcontroller through any on of the downloading tools such as universal programmer. Hence the program should be cross compiled before downloading it into the microcontroller; the keil compiler comes into act at this place. Keil Software provides us with software development tools for the 8051 family of microcontrollers. With these tools, we can generate embedded applications for the multitude of 8051 derivatives. Keil provides following tools for 8051 family development.

1. C51 Optimizing C Cross Compiler,

2. A51 Macro Assembler,

3. 8051 Utilities (linker, object file converter, library manager),

4. Source-Level Debugger/Simulator,

5. $\mu$ Vision for Windows Integrated Development Environment.

The keil 8051 tool kit includes three main tools, assembler, compiler and linker.

- An assembler is used to assemble the 8051 assembly program

- A compiler is used to compile the $\mathrm{C}$ source code into an object file

- A linker is used to create an absolute object module suitable for the in-circuit emulator.

A project development cycle: - these are the steps to develop a project using keil

1. Create source files in $\mathrm{C}$ or assembly.

2. Compile or assemble source files.

3. Correct errors in source files.

4. Link object files from compiler and assembler.

5. Test linked application.

Programming and Downloading the Hex file to the microcontroller,

1. Select the microcontroller in which the hex file have to be downloaded. In this project AT89S52 is selected from the 8051 microcontroller family.

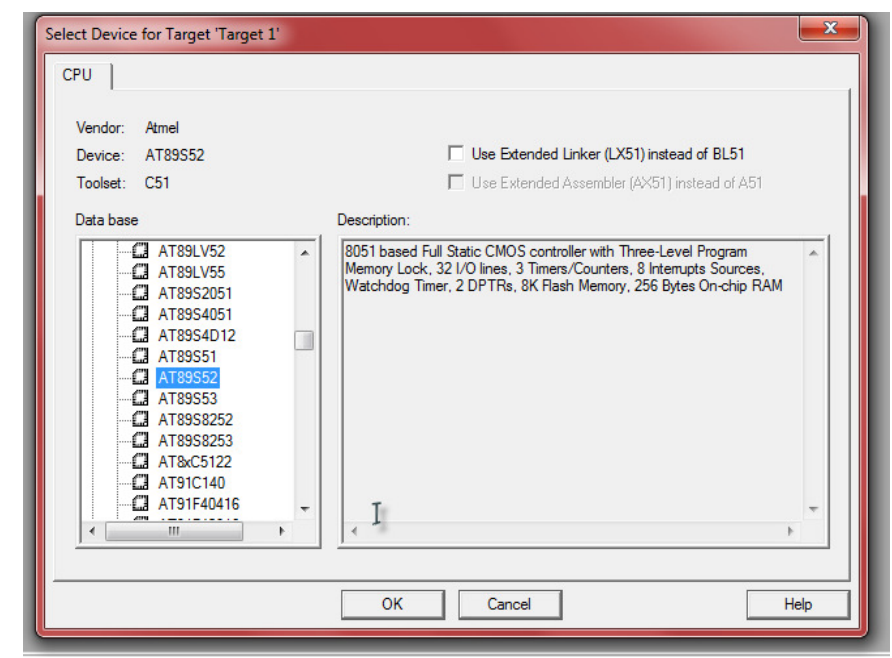


2. Add the source files i.e. the .C files to the project.

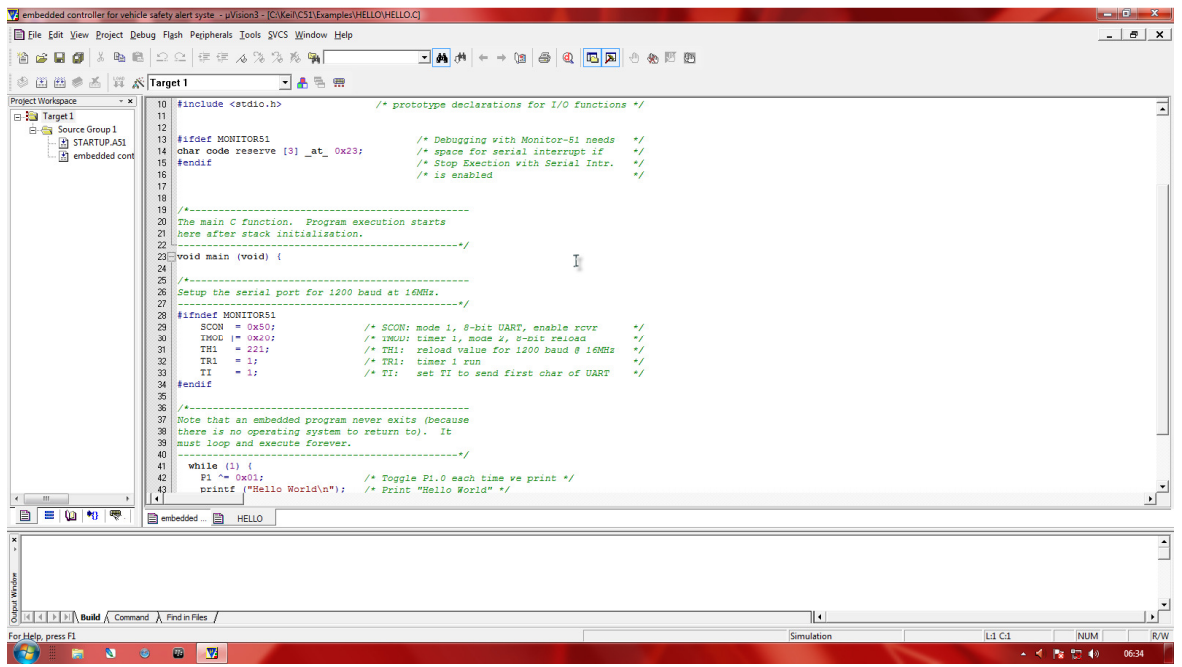

3. Select the option create hex file to create the hex file while running the program.

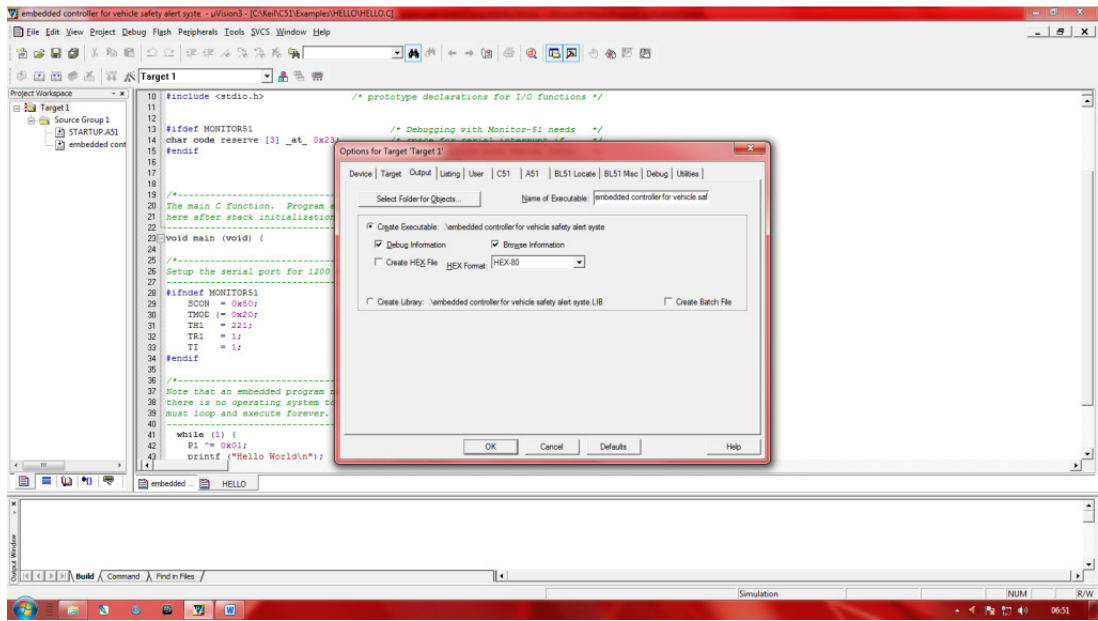

4. Download hex files to the respective microcontroller.

\section{IMPLEMENTATION}

An embedded controller is implemented by using two set of modules, i.e. the obstacle sensing module and the gas detection module. The obstacle detection module is mounted in front of the vehicle such that it searches for the static obstacles. The IR transmitter transmits the IR signals continuously to the maximum level of $3 \mathrm{ft}$. when the IR signals are reflected back by an object then the signal is received by the IR receiver, thus the module senses the obstacle. It then sends the corresponding signals to the microcontroller such that an alarm is provoked by 
International Journal of Computer Science \& Information Technology (IJCSIT) Vol 4, No 2, April 2012

the microcontroller as the first measure and stops the vehicle. The data flow diagram of the obstacle sensing module is shown in the fig.9.

In the same way the gas detection module which is placed inside the vehicle continuously senses the presence of gas in the vehicle cabin. When excess levels of toxic gases such as CO greater than 20ppm, LPG higher than 10,000ppm and the presence of alcohol are found, then the corresponding signals are sent to the microcontroller which in turn provokes an alarm to alert the passengers in the vehicle. And in turn commands the GSM module to send an SMS to the authorised user about the alarming situation inside the vehicle using the AT+CMGS command. The flow diagram of the gas sensing module is shown in the fig.11. The GSM module is connected to the microcontroller by the means of RS232 cable as the communication between the GSM and the microcontroller is synchronised through the MAX232. The system architecture of the proposed system is shown in the fig. 10 .

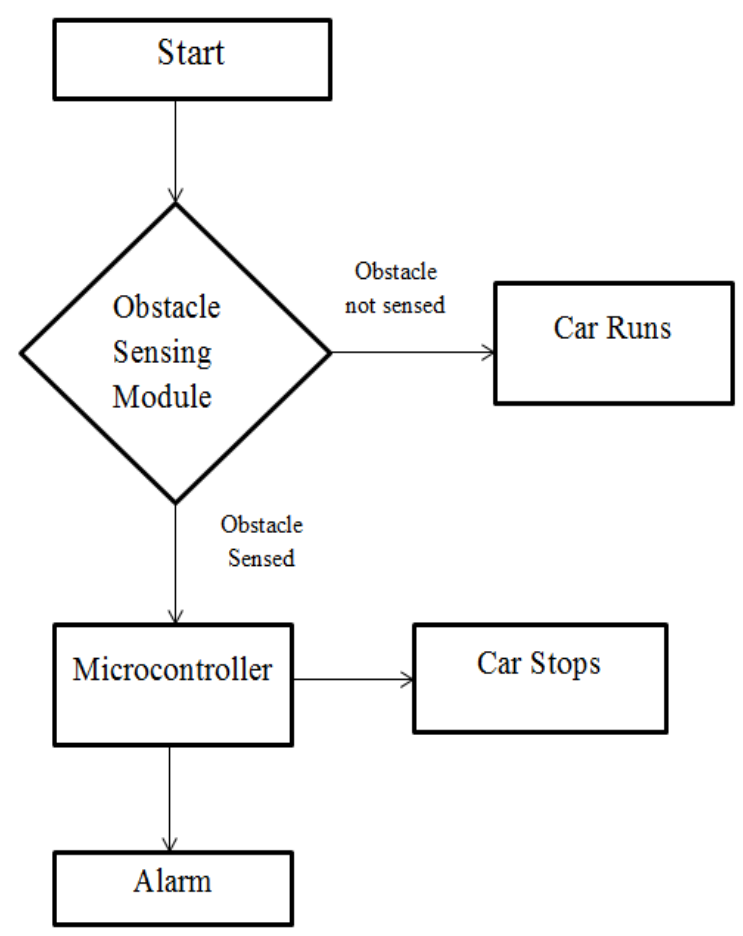

Fig.9 Flow Chart for Working of Obstacle Sensing Module 
International Journal of Computer Science \& Information Technology (IJCSIT) Vol 4, No 2, April 2012

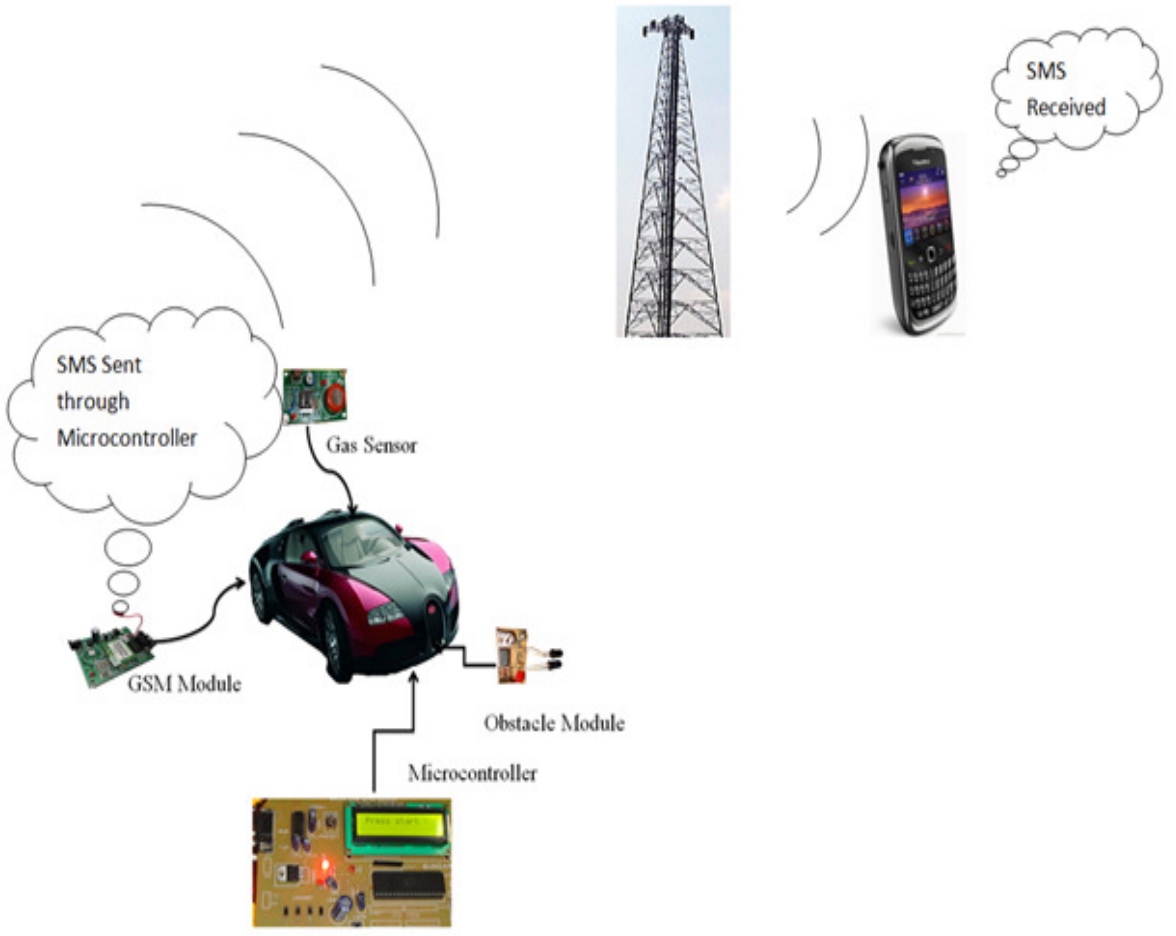

Fig.10 System Architecture of the Proposed System

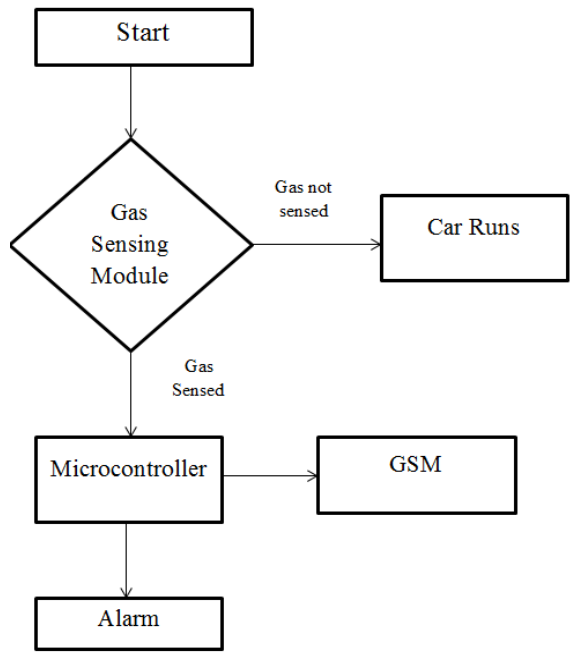

Fig.11.Flow Chart of working of Gas Sensing Module 
International Journal of Computer Science \& Information Technology (IJCSIT) Vol 4, No 2, April 2012

\section{CONCLUSION}

An embedded system has been developed which senses the toxic gases inside the vehicle and alerts the person inside the vehicle, the system also send an alert message to the authorised user through GSM such that remedy measures could be easily taken for the passengers inside a vehicle. The developed system also senses the obstacles in front of the vehicle and so that the accidents due to static obstacles could be avoided. The proposed system has been developed in a special motive that should protect not only the passengers inside the vehicle but also the persons around it such as pedestrians, and to prevent collision of vehicles with any other vehicle or obstacles such as trees. The developed system is greatly helpful to avoid accidents which happen during the night time. The distance between the vehicle and the static obstacle supported by the IR sensor is three feet, and in future the distance could be increased by replacing the IR sensor with the ultrasonic sensor. This system could be further enhanced with future technologies to provide further more safety and security to the vehicle systems.

\section{REFERENCE}

[1]. Hibikino, Wakamatsu, Kitakyushu, “Accident Prevention System Based On Semantic Network Jian-Xiong Yang, JunzoWatada”, Proceedings of the Seventh International Conference on Machine Learning and Cybernetics, Kunming, 12-15 July 2008, pp.: 3738-3743.

[2]. P L Needham of VDO Kienzle UK Ltd., "Collision Prevention: The Role of an Accident Data Recorder (ADR)" in the ADASOl: International Conference on Advanced Driver Assistance Systems September 2001.

[3]. BaoRong Chang, Chung-Ping Young, Hsiu Fen Tsai, and Jian-Jr Lin, from National Taitung University, Taiwan, on "Applying Embedded Hybrid ANFIS/Quantum-Tuned BPNN Prediction to Collision Warning System for Motor Vehicle Safety”, Eighth International Conference on Intelligent Systems Design and Applications 2008.

[4]. Kai-Tai Song and Chih-Chieh Yang, of National Chiao Tung University, Taiwan, "Front Vehicle Tracking Using Scene Analysis", Proceedings of the IEEE International Conference on Mechatronics \& Automation 2005.

[5]. PadminiKumari j, S.S. Dorle, A.G. KeskarMegha, B. Chakole, "Micro-controlled based Vehicle Safety System Using Dedicated Short Range Communications (DSRC)”, Second International Conference on Emerging Trends in Engineering and Technology, ICETET-09.

[6]. Soichi Kubota, Yoshiharu Okamoto, Hideo Oda of National Institute of Information and Communication Technology (NICT), Japan, "Safety Driving Support System Using REID for Prevention of Pedestrian-involved Accidents", 6th International Conference on ITS Telecommunications Proceedings 2006.

[7]. A.R.Ali, E. Imran Zualkernan, and FadiAloul, “A Mobile GPRS-Sensors Array for Air Pollution Monitoring,” vol. 8, pp. 415-422, 2010.

[8]. F.-S. BAI , Y.-L. LIU, "Design of Fault Monitoring Alarm System for Networks Based on GSM SMS,” pp. 45-67, 2010.

[9]. R. Al-Ali, Member, IEEE, Imran Zualkernan, and FadiAloul, Senior Member, IEEE, “A mobile gprs-sensors array for Air pollution monitoring" vol. 6, pp. 410-422, Oct. 2010.

[10]. Da-Jeng Yao, “A gas sensing system for indoor air quality control and polluted environmental monitoring," pp. 11-14, 2009.

[11]. Chen Peijiang, Jiang Xuehua, "Design and Implementation of Remote monitoring system based on GSM,” vol.42, pp.167-175. 2008. 
International Journal of Computer Science \& Information Technology (IJCSIT) Vol 4, No 2, April 2012

[12]. LIU Zhen-ya, WANG Zhen-dong, CHEN Rong, "Intelligent Residential Security Alarm and Remote Control System Based On Single Chip Computer,” vol. 42, pp. 143-166, 2008.

[13]. M.Gao, F.Zhang, andJ.Tian,"Environmental monitoring system with wireless mesh network based on embedded system,"'inProc.5thIEEE Int. Symp. Embedded Computing, 2008, pp. 174179.

[14]. Ruijie Zhang Funjun He, Zhijiang Du and Lining Sun, “ An Intelligent Home Environment Inspecting Robot," vol.42, pp. 140-169, 2007.

[15]. IoanLita, Ion BogdanCioc, Daniel AlexandruVisan, "A New Approach of Automobile Localization System Using GPS and GSM/GPRS Transmission,” vol. 8, pp. 415-422, 2006.

[16]. GoranJurkoviC, Mario iagar, "GSM wireless solutions in distributed embedded systems designed for automatic control" vol.6, pp. 14-18, 2004.

[17]. K. Galatsis, W. Wlodarsla, K. Kalantar-Zadeh and A. Trinchi, "Investigation of gas sensors for vehicle cabin air quality monitoring," vol. 42, pp. 167-175, 2002.

[18]. Gemma Garcia Mandayo, Enrique Castaño, and F. Javier Gracia, "Carbon Monoxide Detector Fabricated on the Basis of a Tin Oxide Novel Doping Method”, VOL. 2, NO. 4, 2002.

[19]. Manabu Kotanit, Takahiko Arimotot, Seiichi Ozawatt, and KenzoAkazawat, "Application of Independent Component Analysis to Detection of Gas Leakage Sound”, 2001.

[20]. K. Galatsis, W. Wlodarski, Y.x. Li and K. Kalantar-zadeh, "Vehicle cabin air quality monitor using gas sensors for improved safety,” pp. 143-164, 2000.

[21]. S.Nagata, M.Matsuno, K.Ogino and H.Takashima, "Carbon Monoxide Detector Unit” 2000.

[22]. Raj Kamal, "Embedded system Architecture programming and design" TATA McGraw Hill. 